\title{
Solving the $k$-center Problem Efficiently with a Dominating Set Algorithm
}

\author{
Jurij Mihelič and Borut Robič \\ Faculty of Computer and Information Science, University of Ljubljana, Slovenia
}

\begin{abstract}
We present a polynomial time heuristic algorithm for the minimum dominating set problem. The algorithm can readily be used for solving the minimum $\alpha$-all-neighbor dominating set problem and the minimum set cover problem. We apply the algorithm in heuristic solving the minimum $k$-center problem in polynomial time. Using a standard set of 40 test problems we experimentally show that our $k$-center algorithm performs much better than other well-known heuristics and is competitive with the best known (non-polynomial time) algorithms for solving the $k$-center problem in terms of average quality and deviation of the results as well as the execution time.
\end{abstract}

Keywords: combinatorial problems, graph algorithms, performance evaluation, facility location, $k$-center, dominating set.

\section{Introduction}

Problems of finding the best location of facilities in networks or graphs abound in practical situations, such as determining locations for fabrication, assembly plants, warehouses, airline crew scheduling or tool selection $[3,15]$. One of the well known facility location problems is the vertex $k$-center problem, where given $n$ cities and distances between all pairs of cities, the aim is to choose $k$ cities (called centers) so that the largest distance of any city to its nearest center is minimal. Formally, the vertex $k$-center is defined as follows.

Definition 1. (Vertex $k$-center problem) Let $G=(V, E)$ be a complete undirected graph with edge costs satisfying the triangle inequality, and $k$ be a positive integer not greater than $|V|$. For any set $S \subseteq V$ and vertex $v \in V$, define $d(v, S)$ to be the length of a shortest edge from $v$ to any vertex in $S$. The problem is to find such a set $S \subseteq V$, where $|S| \leq k$, which minimizes $\max _{v \in V} \bar{d}(v, S)$.

The vertex $k$-center problem is $N P$-hard [6], so exact polynomial algorithm is unlikely to exist. A popular way to solve the $k$-center problem consists of solving a series of minimum set cover problems $[3,5,10,11]$, where one must locate a minimum number of centers such that every vertex in the graph can be reached (i.e. covered) within a given coverage distance. At each step, a threshold for the coverage distance is chosen and the corresponding minimum set cover problem is solved. If the solution contains at most $k$ centers the threshold is decreased, otherwise it is increased. Each of the minimum set cover problems is usually solved using integer linear programming. Minieka [11] was among the first to use this approach. Later, however, more elaborate versions were described, such as Daskin [3], Ellumni et al. [5] as well as Ilhan et al. [10], which applied more efficient integer programming definition of the problem. Another approach was given by Daskin [4] who efficiently used maximum cover problem instead of the minimum set cover problem. A set of completely different approaches to solve the $k$-center problem was given by Mladenović et al. [12], where the tabu search, variable neighborhood search, and various greedy methods were used. The greedy method was also applied by Gonzalez [7], Hochbaum and Shmoys [9], and Shmoys [16]. The last three papers describe 2approximation algorithms for the $k$-center problem. These algorithms are the best possible 
in the sense that no $r$-approximation algorithm exists with $r<2$, unless $P=N P$ [8]. (Notice, that when the triangular inequality does not hold no constant approximation ratio algorithm exists, unless $\mathrm{P}=\mathrm{NP}$.)

In this paper we solve the $k$-center problem as a series of a minimum dominating set problems $[8,9,13]$ (as opposed to the series of minimum set cover or maximum cover problems). Given a graph, the minimum dominating set problem asks for a minimum size subset $D$ of vertices, such that every other vertex is adjacent to at least one of vertices in $D$. The main reason that we use this approach is that the minimum dominating set problem can be identified as one of the main subproblems when solving the $k$-center problem. Unfortunately, the minimum dominating set problem is still $N P$-hard [6], as are the minimum set cover problem and maximum cover problem.

In the following section we describe a heuristic algorithm for the dominating set problem. In Section 3 we show how this algorithm can be applied to solve the $k$-center problem. Section 4 briefly gives some other possible applications of the dominating set algorithm. Finally, in Section 5 we present experimental results that we obtained by comparing our algorithm with several other well-known algorithms for the $k$ center problem. For comparison, we used a standard test library and results that were presented in recent literature.

\section{A Dominating Set Algorithm}

In this section we present a heuristic algorithm for solving the minimum dominating set problem. This problem is formally defined as follows.

Definition 2. (Dominating set) Let $G=(V, E)$ be a graph. A set $D \subseteq V$, such that every vertex $v \in V-D$ is adjacent to at least one vertex in $D$, is called dominating set of $G$.

Definition 3. (Minimum dominating set problem) Given a graph, find a dominating set with minimum cardinality.

Let us first briefly present the main ideas of the heuristic algorithm for the minimum dominating set problem (see algorithm in Figure 1).
Input: graph $G=(V, E)$

Output: dominating set $D$

Algorithm:

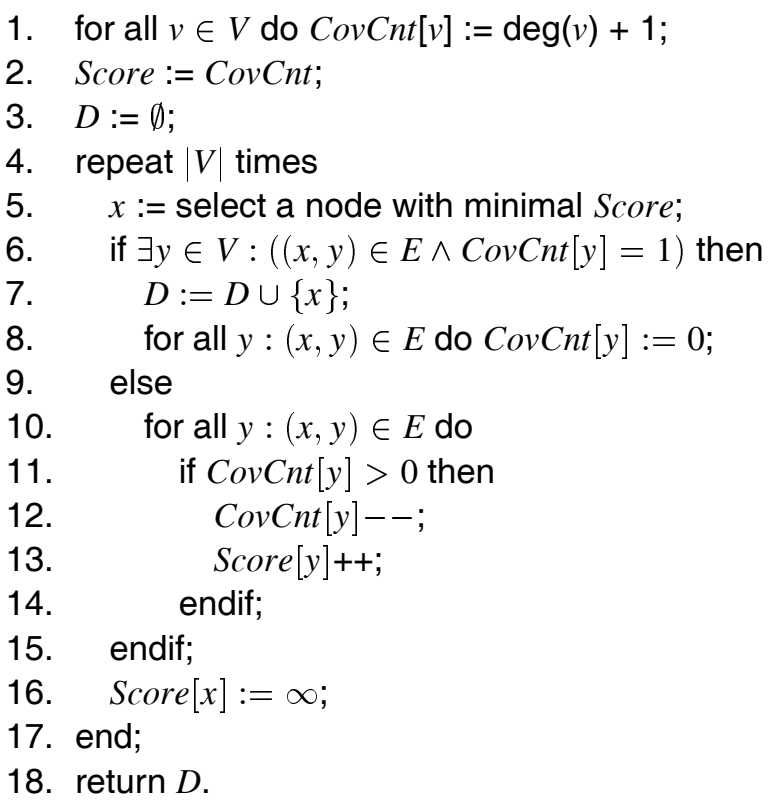

Fig. 1. The dominating set heuristic algorithm.

Initially, the dominating set is empty, $D:=\emptyset$. During the algorithm the set $D$ grows according to the 'lazy' principle, i.e. enlarge $D$ as late as possible. For each vertex $v \in V$ we keep the count $\operatorname{Cov} C n t[v]$, which is the number of times the vertex is covered by the remaining vertices and is initialized to $\operatorname{deg}(v)+1$, where $\operatorname{deg}(v)$ denotes the degree of $v$.

We also estimate the 'importance' of $v$ by applying a special scoring strategy to rank the vertices as potential centers. Then, at each step, a vertex $x$ with minimal score is checked, i.e. if there is an adjacent vertex having cover count 1 , then $x$ is added to $D$; otherwise $x$ is used to decrement cover counts and increment scores of its not yet covered adjacent vertices.

Detailed description. Lines 1-3 are the initialization part of the algorithm. In addition to setting $D$ to empty set, they also initialize two arrays. The first array is CovCnt, where CovCnt $[v]$ is the number of vertices currently covered by (i.e. adjacent to) $v$. We assume that $v$ is adjacent to itself. The second array, called Score, contains scores of vertices. Initially, the score of a vertex equals to its cover count to 
reflect the rule of thumb that vertices with low degrees should be checked first.

Lines 4-17 are the main loop of the algorithm. At each execution of the loop a vertex $x$ with minimal score is selected (line 5). Next, it is checked if $x$ has a neighbor with cover count equal to 1 (line 6). If so, the vertex $x$ is the only possible remaining vertex in the graph that can cover such a neighbor. Hence, $x$ must be added to the current dominating set $D$. Cover counts of all neighbors of $x$ are set to 0 to designate them as covered (lines 7,8). If $x$ has no neighbor with cover count 1 , it is used to improve the current heuristic scoring of those among its neighbors that are still not covered (lines 10-14). To do this, cover counts of $x$ 's neighbors are decremented (because $x$ doesn't cover them anymore) while their scores are incremented (they worth more). At the end of the loop-body the vertex $x$ is designated to be checked by setting Score $[x]$ to infinity.

Correctness. Clearly, the algorithm terminates after $|V|$ executions of the loop body. The algorithm returns a dominating set of the input graph. To see this, consider separately isolated and non-isolated vertices of a graph. First, all the possible isolated vertices $($ Score $[x]=1)$ of the graph are checked and added $(\operatorname{Cov} \operatorname{Cnt}[x]=$ $1)$ to the dominating set $D$. After all isolated vertices have been checked, the algorithm continues on non-isolated vertices, i.e. vertices with initial cover count and score greater than 1. Now consider a non-isolated vertex $y$. The vertex will be covered either because one of its neighbors, say $x$, has been added to $D$ (due to some $x$ 's neighbor $z \neq y$ with cover count 1) or because $y$ 's cover count has reached 1 after $\operatorname{deg}(y)$ decrements (because at each step a different vertex $x \in V$ is checked). Thus, every non-isolated vertex will be covered, too. Consequently, all the vertices of the input graph will be covered, so the algorithm in Figure 1 finds a dominating set.

Complexity. Let us write $n=|V|$. The initialization (lines 1-3) takes $O\left(n^{2}\right)$ time. The body of the main loop is executed $n$ times. At each execution the minimal score vertex can be found in $O(n)$ time. The condition (line 6) as well as inner loops (lines 8 and 10-14) can all be evaluated in $O(n)$ time. Thus, the algorithm terminates in $O\left(n^{3}\right)$ time. However, if vertices are always processed in the same order (determined by a list $L$ ), the time complexity can easily be reduced to $O\left(n^{2}\right)$ by using backtracking. More precisely, suppose that the else part (lines 10-14) is to be executed. While processing the vertices (line 10) one can also check if the condition (line 6) is still false. If the condition has become true, loop is broken, the current vertex is remembered as the backtrack vertex and backtracking procedure is started. In this procedure $x$ is added to $D$ and $x$ 's neighbors are processed, i.e. their cover counts are set to 0 and each neighbor's score is decremented if the neighbor is located before the backtrack vertex in $L$. Thus, backtracking gives two separate inner for loops each with time complexity $O(n)$.

Example. Let us demonstrate a simple example on how the dominating set algorithm from Figure 1 works on a network shown in Figure 2.

There are 8 vertices in this network, thus the algorithm will execute in 8 steps. Execution of the algorithm is shown in Table 1. The first

\begin{tabular}{c|c|cccccccc}
$v$ & $S / C$ & $b$ & $c$ & $h$ & $d$ & $a$ & $f$ & $g$ & $e$ \\
\hline \hline$a$ & $4 / 4$ & & $5 / 3$ & & $6 / 2$ & $\infty / 1$ & & $\infty / 0$ & \\
$b$ & $3 / 3$ & $\infty / 2$ & & & & & $\infty / 1$ & & $\infty / 0$ \\
$c$ & $3 / 3$ & & $\infty / 2$ & & & $\infty / 1$ & & $\infty / 0$ & \\
$d$ & $4 / 4$ & & & & $\infty / 3$ & $\infty / 2$ & & $\infty / 0$ & $\infty / 0$ \\
$e$ & $5 / 5$ & $6 / 4$ & & $7 / 3$ & $8 / 2$ & & $9 / 1$ & & $\infty / 0$ \\
$f$ & $4 / 4$ & $5 / 3$ & & $6 / 2$ & & & $\infty / 1$ & & $\infty / 0$ \\
$g$ & $4 / 4$ & & $5 / 3$ & & $6 / 2$ & $7 / 1$ & & $\infty / 0$ & \\
$h$ & $3 / 3$ & & & $\infty / 2$ & & & $\infty / 1$ & & $\infty / 0$
\end{tabular}

Table 1. Steps of the dominating set algorithm. 


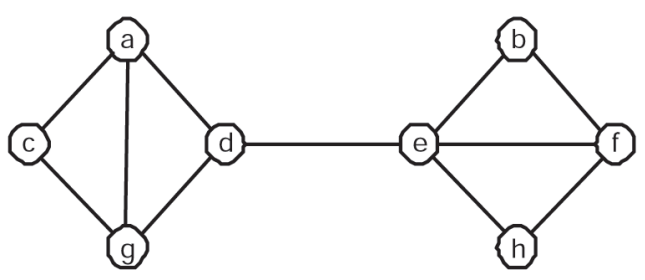

Fig. 2. Example network for dominating set problem.

column lists all of the network vertices. The second column $S / C$ shows the scores and cover counts for each vertex after initialization part of the algorithm. Columns 3-10 give values of score and cover count after each step (only values of processed vertices are shown). The first row contains the vertex that is being processed.

For example, in the third column the first step of the algorithm is shown where vertex $b$ is processed (has minimal score). Vertex $b$ is not added to $D$ because all of its neighbors have cover count greater than 1 . Thus, score is incremented and cover count is decremented, for all $b$ 's neighbors. Score of $b$ is set to $\infty$. In the ninth column of the table vertex $g$ is processed and it must be added to $D$ because at least one of $g$ 's neighbors has cover count equal to 1 (vertices $a, c$ and $g$ ). All cover counts of $g$ 's neighbors are set to 0 to designate them as covered.

\section{Application to the $k$-center Problem}

The algorithm for solving the $k$-center problem uses described dominating set algorithm and parametric pruning, an algorithmic technique presented in $[9,14,17]$, The algorithm is called Scr and is formally described in Figure 3. Initially, edge costs are sorted in nondecreasing order. For each edge cost $t$ the graph is pruned by removing edges with cost greater than $t$ and the minimum dominating set $C$ is found in the pruned graph (BottleneckGraph). If the size of $C$ is less than or equal to $k$, then $C$ is also the optimal solution of the $k$-center problem. As we already mentioned, the minimum dominating set problem is $N P$-hard. To obtain efficient algorithm for the $k$-center problem we applied our algorithm in Figure 1 and the parametric pruning technique from Figure 3.

Complexity. The first phase of the Scr algorithm, which is sorting of the edge costs, takes $O(m \log m)=O\left(n^{2} \log n\right)$ time. The main loop (lines 2-6) takes $O(m)=O\left(n^{2}\right)$ time. Nodes $u$ and $v$ are adjacent in the pruned graph if the distance $d(u, v) \leq c_{i}$, which can directly be used in DominatingSet procedure. Thus, the algorithm Scr has polynomial time complexity $O\left(m n^{3}\right)=O\left(n^{5}\right)$. Notice that by using binary search instead of iterative search (line 2 ), and by using backtracking in the DominatingSet procedure, the time complexity of the $k$-center algorithm can easily be reduced to $O\left(n^{2} \log m\right)=O\left(n^{2} \log n\right)$.

\section{Other Applications}

The algorithm in Figure 1 can easily be extended to solve another version of the dominating set, called minimum $\alpha$-all-neighbor dominating set problem where the aim is to cover every vertex (even centers) with at least $\alpha \geq 1$ vertices. To do this, one simply changes the condition in line 6 of the algorithm. In particular, instead of checking if $\operatorname{Cov} C n t[y]=1$, the equality $\operatorname{Cov} C n t[y]=\alpha$ must be checked. Notice, however, that feasible solution of such a problem may not exist, so one should check at the

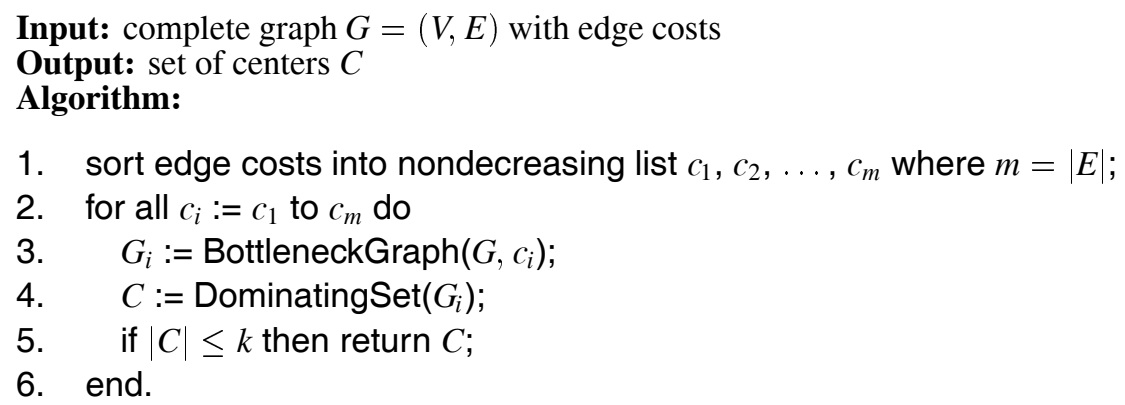

Fig. 3. The algorithm Scr for the $k$-center problem. 
beginning (after line 1) if all cover counts are $\geq \alpha$. Further, if applying this algorithm to the $k$-center algorithm in Figure 3, we obtain the algorithm for minimum $\alpha$-all-neighbor $k$-center problem.

Additionally, one can apply the idea of vertex scores and cover counts to solve the well-known minimum set cover problem where, given a family $\mathcal{F}=\left\{S_{1}, S_{2}, \ldots, S_{N}\right\}$ of subsets of a finite set $X=\left\{x_{1}, x_{2}, \ldots, x_{n}\right\}$, such that $X=\cup_{S \in \mathcal{F}} S$, the aim is to find a minimum cardinality subset $\mathcal{C} \subseteq \mathcal{F}$ such that every element of $X$ belongs to at least one set in $\mathcal{C}$. To find a minimum set cover the algorithm in Figure 1 is changed to track cover count for each element $x \in X$ and score for every set $S \in \mathcal{F}$. Variable $x$ in the algorithm now represents a set with minimal score, the variable $y$ represents elements of $X$, while the relation $(x, y) \in E$ stands for $y \in x$. Line 13 must also be changed to increment the score of all sets containing $y$.

\section{Experimental Results}

In this section we briefly describe several implemented and tested algorithms for the $k$-center problem. After describing the testing environment we discuss experimental results obtained on a set of 40 standard test problems [1].

Implemented algorithms. A very simple heuristic to solve the $k$-center problem is the pure greedy method, where centers are located consecutively so that the objective function is each time reduced as much as possible. We tested three approaches to locate the first center: (1) random, (2) take the solution of the 1-center algorithm, or (3) start from the vertex that offers the best solution (here $n$ runs have to be made). These approaches are called random $(\mathrm{GrR})$, 1-center $(\mathrm{Gr} 1)$, and plus $(\mathrm{Gr}+)$ version, respectively.

Another greedy heuristic for the $k$-center problem was described by Gonzalez [7] and Dyer and Frieze [2]. They were able to prove the approximation factor of 2 . At each step of Gonzalez algorithm the vertex that is the farthest from the current set of centers is added to this set. We implemented and tested the random (GonR), 1-center (Gon1) and plus (Gon+) version of Gonzalez algorithm.

Shmoys [16] briefly described a 2-approximation algorithm for the decision version of $k$-center problem. Here, radius $r$ is given and the aim is to decide if there are $k$ vertices so that the coverage distance from these vertices is at most $r$. The algorithm repeatedly chooses one of the remaining vertices $v$, adds it to the partial solution and deletes all the vertices whose distance to $v$ is at most $2 r$. At the end, if the size of the solution exceeds $k$, the algorithm outputs "no", otherwise "yes". Based on this we implemented two optimization versions of this algorithm, where either random $(\mathrm{ShR})$ vertex or vertex with maximum degree $(\mathrm{ShD})$ is chosen at each step.

Hochbaum and Shmoys introduced the algorithmic technique called parametric pruning for solving the $k$-center problem $[9,17]$. Initially, edge costs are sorted in nondecreasing order and for each edge cost $t$ a maximal independent set in the square of the pruned graph is found.

\begin{tabular}{c||c|c|l}
\multicolumn{1}{c||}{} & \multicolumn{2}{c|}{ Approximation factor } & \\
Algorithm & average & deviation & Description \\
\hline \hline GrR & 1.697 & 0.559 & Pure greedy first random \\
Gr1 & 1.675 & 0.570 & Pure greedy first 1-center \\
Gr+ & 1.512 & 0.550 & Pure greedy plus \\
GonR & 1.495 & 0.130 & Gonzalez first random \\
HS & 1.462 & 0.177 & Hochbaum-Shmoys \\
ShR & 1.432 & 0.112 & Shmoys random \\
Gon1 & 1.398 & 0.128 & Gonzalez first 1-center \\
ShD & 1.343 & 0.105 & Shmoys degree \\
Gon+ & 1.317 & 0.139 & Gonzalez plus \\
Scr & $\mathbf{1 . 0 5 8}$ & $\mathbf{0 . 0 4 3}$ & Scoring heuristic
\end{tabular}

Table 2. The quality of the Scr algorithm. 
If the set contains no more than $k$ vertices, it is returned as the solution of the $k$-center problem; otherwise, the threshold $t$ is increased. The authors have proved that this algorithm (HS) returns 2-approximate solutions. The mentioned algorithms are listed in Table 2. Notice that we also included our algorithm ( $\mathrm{Scr}$ ).

Testing environment. For testing the described algorithms we used the standard ORLibrary, which contains 40 test graphs. These graphs contain from 100 to 900 vertices while $k$ ranges from 5 to 90 . Originally, the library was designed for testing $k$-median problems [1], but it has become also a standard testing tool for the $k$-center problem because the optimal solutions are known. They had been obtained mostly with integer programming approaches $[4,5,10]$, tabu search or variable neighbourhood search [12]. (The preprocessing runs the all shortest paths algorithm with time complexity $O\left(n^{3}\right)$ ).

We implemented all the algorithms from Table 2 in Borland Delphi 7.0 and tested on a computer with Intel processor running at $1700 \mathrm{MHz}$ with $512 \mathrm{MB}$ of system memory.

Experimental results. Although our primary aim was to compare the quality of the solutions, let us mention that Gonzalez's algorithms were the fastest (running below 1 second). The pure greedy methods were quite fast (about $1.5 \mathrm{sec}$ onds on average), but their execution time was very variable and dependent on the parameter $k$. (Recall, that plus variants run much slower due to the algorithm which tries all vertices for the first center.) The average time of HS was about

\begin{tabular}{c||c|c}
\multicolumn{1}{c||}{} & \multicolumn{2}{c}{ Running time } \\
Algorithm & average & deviation \\
\hline \hline GonR & 0.006 & 0.01 \\
Gon1 & 0.01 & 0.012 \\
ShR & 0.204 & 0.142 \\
ShD & 0.211 & 0.147 \\
GrR & 1.526 & 2.37 \\
Gr1 & 1.565 & 2.37 \\
Gon+ & 2.407 & 3.85 \\
Scr & $\mathbf{1 1 . 9 2}$ & $\mathbf{1 0 . 7 7}$ \\
HS & 37.85 & 45 \\
Gr+ & 823 & 1456
\end{tabular}

Table 3. Running times of the algorithms.

38 seconds. Shmoys' variants were also quite fast (below 1 second) and the Scr running time was 12 seconds on average. Average running times of the algorithms are summarized in the following table.

Recall that approximation factor is defined to be the ratio between approximate and optimal solution. Average approximation factors for the algorithms compared are given in Table 3 above. The entries are sorted according to the average quality of the approximate solutions.

Having the smallest average approximation factor, our Scr algorithm performs much better than any other heuristic approach. In addition, this algorithm exhibits the smallest deviation of the approximation factor. Figure 4 also shows that there is a considerable quality leap between Scr algorithm and Gon+, which is the second best of these algorithms.

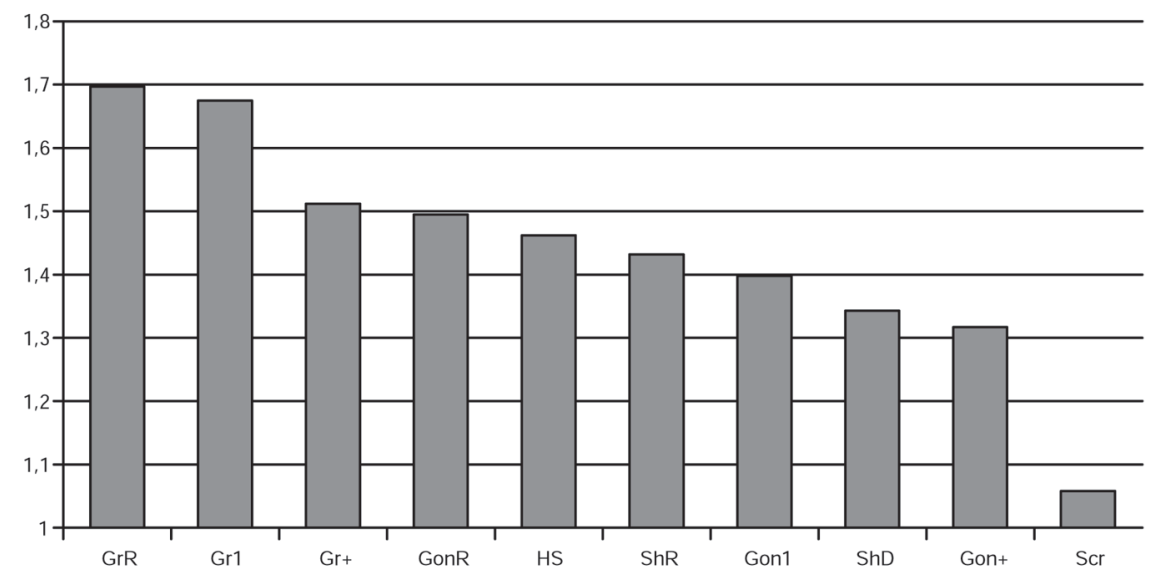

Fig. 4. The quality of algorithms. 


\begin{tabular}{|c|c|c|c|c|c|c|c|c|c|c|c|c|c|}
\hline \# & $\mathrm{n}$ & $\mathrm{k}$ & Opt & GrR & Gr 1 & $\mathrm{GrP}$ & Gon & Gon1 & Gon+ & HS & ShR & ShD & $\mathrm{Scr}$ \\
\hline 1 & 100 & 5 & 127 & 143 & 133 & 133 & 186 & 162 & 155 & 184 & 188 & 171 & 133 \\
\hline 2 & 100 & 10 & 98 & 117 & 117 & 110 & 131 & 124 & 117 & 160 & 128 & 135 & 109 \\
\hline 3 & 100 & 10 & 93 & 126 & 116 & 106 & 154 & 133 & 124 & 160 & 140 & 120 & 99 \\
\hline 4 & 100 & 20 & 74 & 127 & 127 & 92 & 114 & 99 & 92 & 124 & 109 & 84 & 83 \\
\hline 5 & 100 & 33 & 48 & 87 & 87 & 78 & 71 & 64 & 62 & 77 & 62 & 59 & 48 \\
\hline 6 & 200 & 5 & 84 & 98 & 94 & 89 & 138 & 99 & 98 & 126 & 138 & 106 & 90 \\
\hline 7 & 200 & 10 & 64 & 78 & 79 & 77 & 96 & 87 & 85 & 90 & 88 & 90 & 70 \\
\hline 8 & 200 & 20 & 55 & 72 & 72 & 72 & 82 & 72 & 71 & 84 & 74 & 68 & 60 \\
\hline 9 & 200 & 40 & 37 & 73 & 73 & 63 & 57 & 51 & 49 & 62 & 50 & 52 & 38 \\
\hline 10 & 200 & 67 & 20 & 44 & 44 & 38 & 31 & 29 & 29 & 32 & 28 & 28 & 20 \\
\hline 11 & 300 & 5 & 59 & 68 & 67 & 61 & 73 & 68 & 68 & 82 & 73 & 74 & 60 \\
\hline 12 & 300 & 10 & 51 & 62 & 72 & 56 & 71 & 70 & 66 & 78 & 74 & 70 & 53 \\
\hline 13 & 300 & 30 & 35 & 64 & 64 & 52 & 59 & 51 & 49 & 60 & 54 & 52 & 38 \\
\hline 14 & 300 & 60 & 26 & 60 & 60 & 46 & 40 & 36 & 36 & 44 & 36 & 34 & 27 \\
\hline 15 & 300 & 100 & 18 & 42 & 42 & 40 & 25 & 25 & 23 & 30 & 22 & 20 & 18 \\
\hline 16 & 400 & 5 & 47 & 52 & 51 & 47 & 84 & 55 & 52 & 64 & 83 & 58 & 48 \\
\hline 17 & 400 & 10 & 39 & 50 & 50 & 43 & 56 & 51 & 48 & 56 & 56 & 52 & 41 \\
\hline 18 & 400 & 40 & 28 & 50 & 50 & 42 & 44 & 41 & 39 & 46 & 40 & 38 & 31 \\
\hline 19 & 400 & 80 & 18 & 40 & 40 & 31 & 28 & 28 & 27 & 30 & 26 & 24 & 20 \\
\hline 20 & 400 & 133 & 13 & 32 & 32 & 32 & 19 & 19 & 17 & 22 & 18 & 16 & 14 \\
\hline 21 & 500 & 5 & 40 & 48 & 48 & 42 & 53 & 51 & 45 & 52 & 53 & 45 & 40 \\
\hline 22 & 500 & 10 & 38 & 48 & 49 & 43 & 56 & 54 & 47 & 54 & 54 & 48 & 41 \\
\hline 23 & 500 & 50 & 22 & 41 & 41 & 35 & 34 & 33 & 32 & 36 & 32 & 30 & 24 \\
\hline 24 & 500 & 100 & 15 & 35 & 35 & 32 & 23 & 23 & 21 & 24 & 22 & 20 & 17 \\
\hline 25 & 500 & 167 & 11 & 27 & 27 & 27 & 15 & 15 & 15 & 18 & 16 & 14 & 11 \\
\hline 26 & 600 & 5 & 38 & 44 & 43 & 39 & 50 & 47 & 43 & 52 & 50 & 52 & 41 \\
\hline 27 & 600 & 10 & 32 & 37 & 39 & 35 & 43 & 42 & 55 & 42 & 44 & 44 & 33 \\
\hline 28 & 600 & 60 & 18 & 33 & 33 & 27 & 28 & 28 & 25 & 28 & 28 & 28 & 20 \\
\hline 29 & 600 & 120 & 13 & 34 & 36 & 34 & 19 & 19 & 18 & 22 & 18 & 18 & 13 \\
\hline 30 & 600 & 200 & 9 & 29 & 29 & 29 & 14 & 14 & 13 & 16 & 12 & 12 & 10 \\
\hline 31 & 700 & 5 & 30 & 35 & 34 & 31 & 42 & 38 & 36 & 40 & 42 & 44 & 30 \\
\hline 32 & 700 & 10 & 29 & 35 & 35 & 32 & 45 & 43 & 37 & 40 & 44 & 40 & 31 \\
\hline 33 & 700 & 70 & 15 & 32 & 26 & 24 & 26 & 25 & 23 & 26 & 24 & 22 & 17 \\
\hline 34 & 700 & 140 & 11 & 30 & 30 & 27 & 17 & 17 & 16 & 18 & 16 & 16 & 11 \\
\hline 35 & 800 & 5 & 30 & 37 & 32 & 31 & 38 & 37 & 34 & 40 & 38 & 38 & 32 \\
\hline 36 & 800 & 10 & 27 & 34 & 34 & 30 & 41 & 41 & 34 & 38 & 42 & 38 & 28 \\
\hline 37 & 800 & 80 & 15 & 26 & 26 & 26 & 25 & 24 & 23 & 24 & 22 & 22 & 16 \\
\hline 38 & 900 & 5 & 29 & 42 & 35 & 31 & 36 & 38 & 31 & 38 & 40 & 38 & 29 \\
\hline 39 & 900 & 10 & 23 & 27 & 28 & 25 & 35 & 35 & 28 & 32 & 36 & 34 & 24 \\
\hline 40 & 900 & 90 & 13 & 25 & 22 & 22 & 21 & 20 & 19 & 22 & 20 & 20 & 14 \\
\hline
\end{tabular}

Table 4. Experimental results: \# is the graph number; $n$ represents the number of vertices; $k$ is the number of centers; Opt is the optimal result; other columns contain results obtained with corresponding algorithms.

The results for particular algorithms and problems are given in Table 4. Pure greedy algorithms were the worst, while only slightly better results were obtained with the plus version. By examining Table 4 we found out that the quality of the pure greedy method strongly depends on the parameter $k$ and improves for low values of $k$. Gonzalez algorithms are very fast. The best variant of the Gonzalez algorithm is the plus variant, yet it still returns results that are about $32 \%$ above the optimal. The algorithms HS, $\mathrm{ShR}, \mathrm{ShD}$ exploit very similar problem properties, and return similar results. However, the algorithm ShD proved to be the best among them. 
The algorithm Scr achieved better results than any other of the implemented algorithms and is also quite competitive with integer programming and metaheuristic approaches. In particular, the solutions of Scr heuristic are on average only $6 \%$ above the optimal.

\section{Conclusions}

In this paper we have presented a polynomial time heuristic algorithm for the minimum dominating set problem, which uses the so-called scoring technique. We applied this algorithm to efficiently solve the $k$-center problem. Then we experimentally evaluated our algorithm and several other well-known heuristic algorithms for the $k$-center problem. To do this, we used the standard set of 40 test problems. The experimental results show that our algorithm is comparable with the best known algorithms for solving the $k$-center problem $[4,5,10]$. These algorithms were able to solve quickly enough each of the forty test problems to optimality. Since they rely on the integer programming, it would be interesting to see how they perform on larger test instances. We have also found that, surprisingly, the performance of the well-known 2-approximation algorithm HS by Hochbaum and Shmoys [9] was quite unsatisfactory, as was noticed also by Mladenović [12]. The same is true for all the tested approximation algorithms. These are all three versions of Gonzalez algorithm (Gon, Gon1, Gon+) and both Shmoys algorithms (ShR, ShD). The pure greedy approach is the worst among all and if, for any reason, it is used, we suggest using it only for small values of $k$.

Finally, in the paper we have shown that our heuristic algorithm for solving the minimum dominating set problem can easily be adapted to solve several other important combinatorial optimization problems. Among these are (1) the minimum $\alpha$-all-neighbor dominating set problem, (2) the minimum set cover problem, and (3) the $\alpha$-all-neighbor $k$-center algorithm. An interesting future research would be to experimentally evaluate, for each of these problems, the corresponding adapted algorithm and compare it with the currently best algorithms.

\section{References}

[1] J.E. BEASLEY, A note on solving large $p$-median problems, European J. Oper. Res., 21:270-273, 1985.

[2] M.E. DYER AND A.M. FRIEZE, A simple heuristic for the p-centre problem, Operations Research Letters, $3: 6: 285-288,1985$.

[3] M.S. DASKIN, Network and Discrete Location: Models Algorithms and Applications, Wiley, New York, 1995.

[4] M.S. DASKIN, A new approach to solving the vertex p-center problem to optimality: Algorithm and computational results, Communications of the $\mathrm{Op}$ erations Research Society of Japan, 45:9:428-436, 2000.

[5] S. Elloumi, M. LABBe, AND Y. Pochet, New formulation and resolution method for the $p$-center problem, 2001. http://www.optimizationonline.org/DB_HTML/2001/10/394.html.

[6] M.R. GAREY AND D.S. Johnson, Computers and Intractability: A Guide to the Theory of NPCompleteness, W.H. Freeman and Co., San Francisco, 1979.

[7] T. GonZaLeZ, Clustering to minimize the maximum intercluster distance, Theoretical Computer Science., 38:293-306, 1985.

[8] D.S. Hochbaum, ed., Approximation Algorithms for NP-hard Problems, PWS publishing company, Boston, 1995.

[9] D.S. Hochbaum AND D.B. ShmoYs, A best possible heuristic for the k-center problem, Mathematics of Operations Research, 10:180-184, 1985.

[10] T. IlHAn AND M.C. PinAR, An efficient exact algorithm for the vertex $p$-center problem, 2001. http: //www.optimization-online.org/ DB_HTML $/ 2001 / 09 / 376 . \mathrm{html}$.

[11] E. MINIEKA, The $m$-center problem, SIAM Rev., $12: 138-139,1970$.

[12] N. Mladenović, M. LABbe, ANd P. HANSEN, Solving the p-center problem with tabu search and variable neighborhood search, Networks 42(1):48-64, 2003.

[13] J. MiHELIČ AND B. RoBIČ, Approximation algorithms for $k$-center problem: an experimental evaluation, Proc. OR 2002, Klagenfurt, Austria, 2002.

[14] J. PlesniK, A Heuristic for the $p$-Center Problem in Graphs, Discrete Applied Mathematics 17:263-268, 1987.

[15] Charles S. ReVelle AND H.A. Eiselt, Location analysis: A synthesis and survey, European Journal of Operational Research, 165:1-19, 2005. 
[16] D.B. ShMOYs, Computing near-optimal solutions to combinatorial optimization problems, Technical report, Ithaca, NY 14853, 1995. http://citeseer. $\mathrm{nj}$. nec.com/shmoys 95computing.html.

[17] V. VAZIRANI, Aproximation Algorithms, Springer, 2001.

Received: September, 2005 Accepted: March, 2005

Contact address: Jurij Mihelič Faculty of Computer and Information Science University of Ljubljana Tržaška 25, 10000 Ljubljana, Slovenia e-mail: jurij.mihelic@fri.uni-lj.si Web: http://lalg.fri.uni-lj.si/ jure

JURIJ MIHELIČ received the BSc (2001) and MSc (2004) degrees in computer science from the University of Ljubljana, Slovenia. He is presently working towards his $\mathrm{PhD}$ at the Faculty of Computer and Information Science, University of Ljubljana. His fields of interests are facility location, combinatorial optimization and approximation algorithms.

BORUT RoBiČ is Professor of computer science at the Faculty of Computer and Information Science, University of Ljubljana, Slovenia. Following completion of his B.Sc. (1984), M.Sc. (1987), and D.Sc. (1993) in computer science at the University of Ljubljana, he held positions as a researcher in the Department of computer science at the Jozef Stefan Institute, and as a Partridge visiting fellow at the University of Cambridge, UK, before joining University of Ljubljana in 1997. His research focuses on algorithms, complexity and parallel computing, with specific interest in approximation and probabilistic algorithms. 